EESTI NSV TEADUSTE AKADEEMIA TOIMETISÉD, 29. KOIDE

FUOSIKA * MATEMAATIKA, 1980, NR. 2

ИЗВЕСТИЯ АКАДЕМИИ НАУК ЭСТОНСКОП ССР. ТОМ 29

ФИЗИКА * МАТЕМАТИКА, 1980, № 2

удК $536.48: 537.228$

B. КОРРОВИТС, М. ТРУММАЛ

\title{
АДИАБАТИЧЕСКИЙ ЭЛЕКТРОКАЛОРИЧЕСКИЙ ЭФФЕКТ В НЕКОТОРЫХ ПАРАЭЛЕКТРИЧЕСКИХ СИСТЕМАХ
}

(Представил К. К. Ребане)

После открытия в 1965 г. электрокалорического эффекта в параэлектрических щелочногалоидных кристаллах при низких температурах основное внимание в работах по параэлектрикам уделялось исследованию самих примесей. Оказалось, что параэлектрические примеси весьма трудно поддаются описанию из-за меняющейся от образца к образцу симметрии системы. «Классических», в смысле возможности простого моделирования, параэлектрических примесей мало. Примером хорошо изученной системы является $\mathrm{KCl}: \mathrm{Li}^{+}\left[{ }^{1}\right]$. Даже равновесную ориентацию примесей, особенно $\mathrm{CN}^{-}$, не удавалось долгое время определить однозначно. Большой разброс наблюдается в значениях электрического дипольного момента $\mu$, параметра туннельного расщепления $\Delta$ и других параметров примесей при измерении их по разным методикам $\left[{ }^{2}\right]$. До сих пор нет удовлетворительной модели конкретных примесей.

Ниже вычисляется величина адиабатического электрокалорического эффекта (АЭЭ) параэлектрического кристалла для выявления роли неучтенных моделью факторов путем сравнения теоретических данных с экспериментальными. Для определенных интервалов внешнего электрического поля и температур получены приближенные выражения для величины АЭЭ.

1. Присутствие параэлектрических примесей в виде идеального дипольного газа с дипольным моментом $\vec{\mu} \|\langle 100\rangle$ в кубическом кристалле при туннелировании между эквивалентными состояниями с поворо-

том $\vec{\mu}$ только на $90^{\circ}$ приводит к расщеплению шестикратно вырожденного состояния. Во внешнем электрическом поле происходит дальнейшее расщепление. Из полученного энергетического спектра $W(E)$ через статистическую сумму $Z$ можно вычислить дипольную энтропию $N$ диполей при ориентации внешнего электрнческого поля $\vec{E} \|[100]\left[{ }^{3}\right]$ :

$$
S_{100} / N k=\ln Z_{100}-\left(\beta / Z_{100}\right) \sum_{i=1}^{4} A_{i} \exp A_{i}
$$

где $\quad Z_{100}=2+\sum_{i=1}^{4} \exp \beta A_{i}, \quad A_{1}=-\Delta, \quad A_{2}=2 a, \quad A_{3}=\Delta / 2+b-a$, $A_{4}=\Delta / 2-a-b, \quad a=\delta^{2} \Delta / 2\left(2 \Delta^{2}+\delta^{2}\right), \quad b=\sqrt{9 / 4 \Delta^{2}+\delta^{2}}, \quad \delta=\mu E, \quad \beta=(k T)^{-1}$, $k$ - постоянная Больцмана. 
В сильном электрическом поле эксперименты проводятся с кристаллами, у которых электродами покрыта только часть поверхности. Дипольная энтропия всего кристалла имеет вид

$$
S=f S_{100}+(1-f) S_{0},
$$

где $S_{0}$ - энтропия примеси только в кристаллическом поле, получается из $S_{100}$ при $E=0 ; f-$ коэффициент покрытости, т. е. отношение площади электродов к площади кристалла.

Из выражения энтропии (1) можно получить точную величину АЭЭ решением уравнения

$$
S\left(0, T_{f}\right)=S\left(E, T_{i}\right) .
$$

Здесь $S(E, T)$ является суммой дипольной и решеточной энтропии, причем $S_{L}=A T^{3} / 3$, где $A-$ коэффициент, входящий в решеточную теплоемкость $C_{V}{ }^{L}=A T^{3} ; T_{i}, T_{f}-$ начальная и конечная температуры при адиабатической деполяризации образца.

На рис. 1 показана величина АЭЭ, $\Delta T=T_{i}-T_{f}$, полученная для системы $\mathrm{KCl}_{1}: \mathrm{OH}^{-}$на ЭВМ решением уравнения (2) методом последовательных приближений. Кривые получены при различных значениях $E$. Некоторое расхождение с экспериментальными данными из $\left[{ }^{4}\right]$, которое увеличивается при понижении температуры и уменьшении электрического поля, объясняется выбором модели, которая не учитывает локальных полей в непосредственном окружении примеси и взаимодействия примесей между собой - факторов, влияющих на движение примеси и на ориентацию ее при адиабатической деполяризации.

Локальные поля описываются в $\mathrm{KCl}: \mathrm{OH}^{-}$распределением Гаусса с полушириной $\Gamma=3 \kappa B \cdot c M^{-1}\left[{ }^{5}\right]$. Поскольку в случае $3 \kappa B \cdot c M^{-1}$ $\mu E / k=0,3 \mathrm{~K}$, понятно, отчего во внешнем поле $\mathrm{c}: 1,5$ и $2,4 \kappa B \cdot c \mathrm{~m}^{-1}$ при $T \lesssim 0,5 \mathrm{~K}$ наблюдается значительное расхождение расчетных и экспериментальных кривых.

Из рис. 2 виднө, что $\Delta T$ в $\mathrm{RbCl}: \mathrm{CN}^{-}$, рассчитанная по модели диполей с $\vec{\mu} \|\langle 100\rangle$, ближе к экспериментальной кривой, чем рассчитанная по модели диполей с $\vec{\mu} \|\langle 111\rangle\left[{ }^{6}\right]$, особенно в высокотемпературной области кривой при $35 \kappa B \cdot c k^{-1}$. В этом поле $\mu E / k=0,25 \mathrm{~K}$, что и создает неопределенность в вычислениях при $T \leqslant 0,2 \mathrm{~K}$. Наша модель не учитывает эластичного взаимодействия диполя $\mathrm{CN}^{-}$с решеткой, а это может оказывать при низких температурах значительное влияние на ориентацию диполя. Если предположить, что в кристалле имеется поле напряжений $\sigma \simeq 10^{6} \partial u н \cdot c M^{-2}$, то вследствие довольно большого эластичного дипольного момента у $\mathrm{RbCl}: \mathrm{CN}^{-}$получается $p_{\lambda}=$ $=1,2 \cdot 10^{-23} \mathrm{~cm}^{3}, p_{\lambda} \sigma / k=0,2 \mathrm{~K}$. Это является одним из существенных препятствий при определении ориентации примеси $\mathrm{CN}^{-}$. Измерения Керр-эффекта $\underset{\rightarrow}{\left[{ }^{7}\right]}$ показали, что $\vec{\mu} \|\langle 111\rangle$. Можно полагать, что вопрос об ориентации $\mu$ в дипольных системах с $\mathrm{CN}^{-}$(см., капр., $\left.\left[{ }^{2}\right]\right)$, теперь решен.

Используемая нами для описания парањлектрических примесей

модель с $\vec{\mu} \|\langle 100\rangle$ вполне удовлетворительно согласуется с экспериментальными данными по АЭЭ, она применялась и для объяснения экспериментов по изотермическому электрокалорическому эффекту $\left[{ }^{3}\right]$. Ввиду того, что в параэлектрических системах различные взаимодействия приводят к спонтанной поляризации диполей, область приме- 


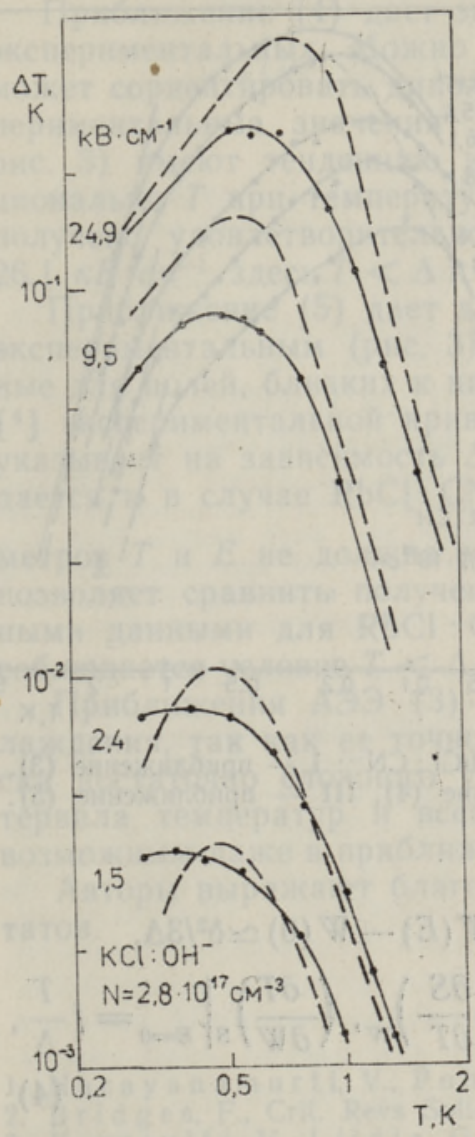

Рис. 1. АЭЭ в $\mathrm{KCl}: \mathrm{OH}^{-}$. Экспериментальные данные (сплошная линия) из $\left[{ }^{4}\right]$.

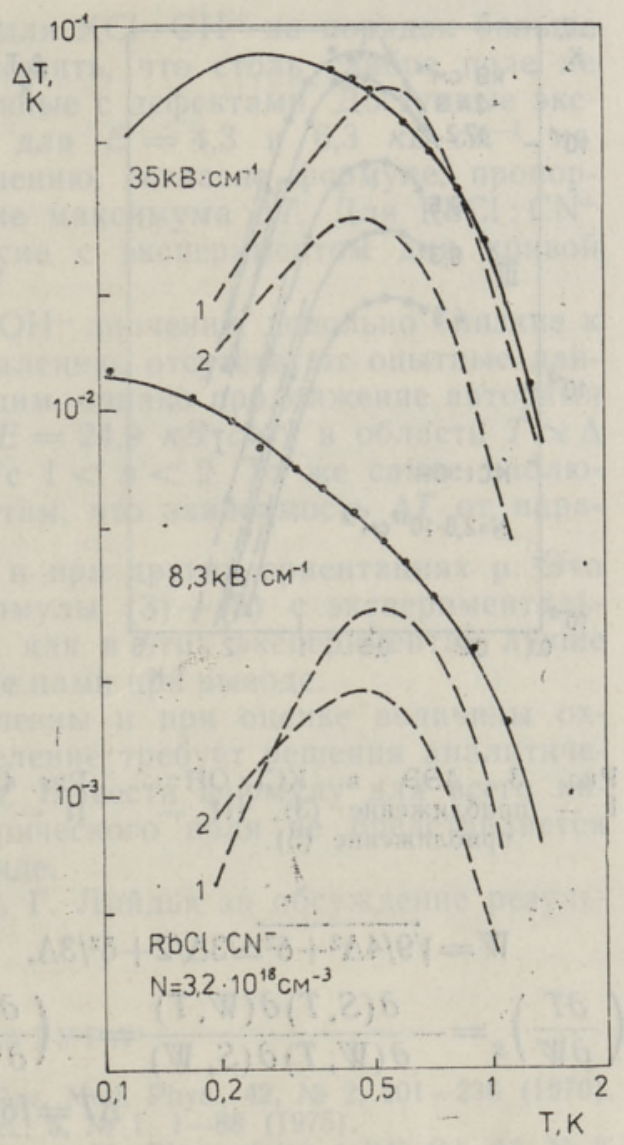

Рис. 2. АЭЭ в $\mathrm{RbCl}: \mathrm{CN}^{-}: 1-$ расчет пө модели $\vec{\mu} \|<100>, 2$ - расчет по модели $\vec{\mu} \|<111>$.

нения данной модели ограничена. Вопрос о дальнейшем ее совершенствовании весьма спорный из-за большого количества факторов спонтанной поляризации.

2. Величину АЭЭ $\Delta T$ в системах с $\vec{\mu} \|\langle 100\rangle$ можно представить аналитически в виде приближений уравнения (2) в определенных интервалах значений $E$ и $T$.

I. Приближение высоких температур и слабых полей: $T \gg \Delta$ и $E<\Delta / \mu$. При выводе пренебрегаем членами типа $\Delta / k T$ и членами второго порядка малости в разложении функций уравнения (2) в степенной ряд $\left[{ }^{8}\right]$. Получается формула

$$
\Delta T=f N \mu^{2} E^{2} / 6 A k T^{4} .
$$

II. Приближение низких температур и слабых полей: $T<\Delta$ и $E<\Delta / \mu$.

$$
\left.\Delta T \simeq(\partial T / \partial W)_{S}\right|_{E=0} \Delta W
$$




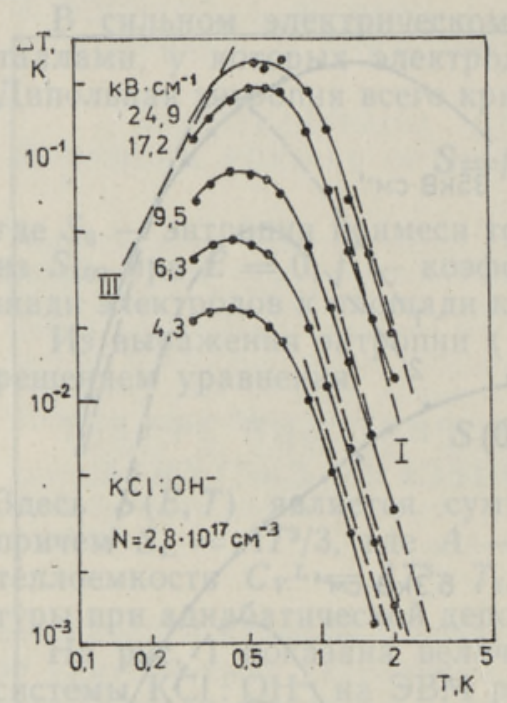

Рис. 3. АЭЭ в $\mathrm{KCl}: \mathrm{OH}^{-}$: I - приближение (3), III приближение (5).

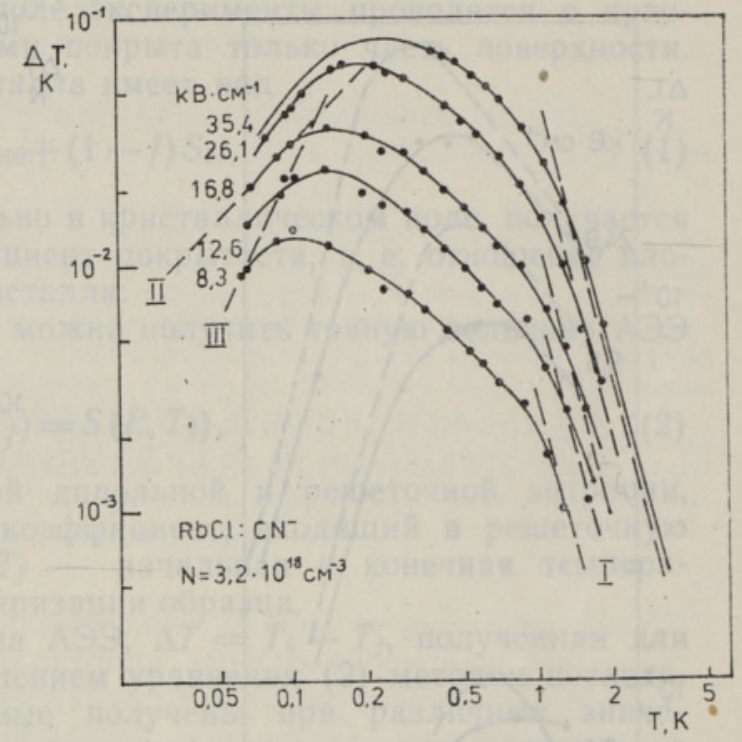

Рис. 4. АЭЭ в $\mathrm{RbCl}: \mathrm{CN}^{-}: \mathrm{I}$ - приближение (3), II - приближение (4), III - приближение (5).

$$
\begin{gathered}
W=\sqrt{9 / 4 \Delta^{2}+\delta^{2}} \simeq 3 \Delta / 2+\delta^{2} / 3 \Delta, \quad \Delta W=W(E)-W(0) \simeq \delta^{2} / 3 \Delta, \\
\left(\frac{\partial T}{\partial W}\right)_{S}=-\frac{\partial(S, T) \partial(W, T)}{\partial(W, T) \partial(S, W)}=-\left(\frac{\partial S}{\partial W}\right)_{T} /\left(\frac{\partial S}{\partial T}\right)_{W},\left.\left(\frac{\partial T}{\partial W}\right)_{S}\right|_{E=0}=f \frac{T}{\Delta}, \\
\Delta T=f \delta^{2} T / 3 \Delta^{2} .
\end{gathered}
$$

III. Приближение низких температур и максимального поля: $T<\Delta$ и $E \gg \Delta / \mu$.

$$
\begin{gathered}
(1-f) S\left(\Delta, T_{i}\right)+f S_{100}\left(E_{\max }, \Delta, T_{i}\right)=S_{0}\left(\Delta, T_{f}\right), \\
S(\Delta, T)=\ln (1+3 \exp (-2 y)+2 \exp (-3 y))+ \\
+\frac{6 y[\exp (-2 y)+\exp (-3 y)]}{1+3 \exp (-2 y)+2 \exp (-3 y)}, \\
S(\Delta, T) \simeq \ln (1+3 \exp (-2 y))+\frac{6 y \exp (-2 y)}{1+3 \exp (-2 y)}, \quad y=\Delta / k T, \\
S\left(T_{f}\right) \simeq S\left(T_{i}\right)-\frac{d}{d y} S(T) \mid T_{t} \frac{d y}{d T} \Delta T, \\
\Delta T=f k T^{2} / 2 \Delta .
\end{gathered}
$$

Полученные приближения (3)-(5) мы сравниваем на рис. 3 и 4 с экспериментальными данными из $\left[{ }^{4}\right]$ в достаточно широком температурном интервале.

Приближение (3), оно, кстати, имеет такой же вид и для других равновесных ориентаций $\vec{\mu}$, хорошо согласуется с приведенными экспериментальными кривыми для кристаллов $\mathrm{KCl}: \mathrm{OH}^{-}$и $\mathrm{RbCl}: \mathrm{CN}^{-}$ при $T>1 \mathrm{~K}$ и $E<\Delta / \mu$ (рис. 3 и 4 ), что удовлетворяет условиям приближения. 
Приближение (4) дает значения для $\mathrm{KCl}: \mathrm{OH}^{-}$на порядок больше экспериментальных. Можно предположить, что столь слабое поле не может сориентировать диполи, связанные с дефектами. Доступные экспериментальные значения (кривые для $E=4,3$ и $6,3 \kappa B \cdot c \mu^{-1}$ на рис. 3) имеют тенденцию к уменьшению, согласно формуле, пропорционально $T$ при температурах, ниже максимума $\Delta T$. Для $\mathrm{RbCl}: \mathrm{CN}^{-}$ получено удовлетворительное согласие с экспериментом для кривой $26,1 \kappa B \cdot c M^{-1}$, здесь $T<\Delta$ и $E \simeq \Delta / \mu$.

Приближение (5) дает для $\mathrm{KCl}: \mathrm{OH}^{-}$значения, довольно близкие к экспериментальным (рис. 3). K сожалению, отсутствуют опытные данные для полей, близких к насыщающим, однако продолжение авторами $\left[{ }^{4}\right]$ экспериментальной кривой для $E=24,9 \kappa B \cdot c M^{-1}$ в область $T \simeq \Delta$ указывает на зависимость $\Delta T \sim T^{n}$ с $1<n<2$. То же самое наблюдается и в случае $\mathrm{RbCl}: \mathrm{CN}^{-}$. Заметим, что зависимость $\Delta T$ от параметров $T$ и $E$ не должна меняться и при других ориентациях $\vec{\mu}$. Это позволяет сравнить полученные формулы (3)-(5) с экспериментальными данными для $\mathrm{RbCl}: \mathrm{CN}^{-}$, так как в этих экспериментах лучше соблюдается условие $T<\Delta$, принятое нами при выводе.

Приближения АЭЭ (3)-(5) полезны и при оценке величины охлаждения, так как ее точное определение требует решения аналитически достаточно сложного уравнения. Вывести формулу для всего интервала температур и всего электрического поля не представляется возможным даже в приближенном виде.

Авторы выражают благодарность Г. Лийдья за обсуждение результатов.

\section{ЛИТЕРАТУРА}

1. N a r a y a n a murti, V., Poh 1, R. O., Rev. Mod. Phys., 42, № 2, 201-236 (1970).

2. B ridges, F., Crit. Revs Solid. State Sci., 5, № 1, 1-88 (1975).

3. Korrovits, V., Liidja, G., Mihkelsoo, V., Phys, status solidi (b), 67, № 2, 695-702 (1975).

4. Pohl, R. O., T a ylor, V. L., Goub a u, W. M., Phys. Rev., 178, № 3, 1431-1436 (1969).

5. Knop, K., Känzi g, W., Phys. Kondens. Materie, 15, № 3, 201-204 (1972).

6. Gom ez, M., B ow en, S. P., Krumhans 1, J. A., Phys. Rev., 153, № 3, 10091024 (1967).

7. D i a z-Gon gor a, A., L üt y, F., Phys. status solidi (b), 86, № 1, 127-136 (1978).

8. Корровит В. Х., Лийдья Г. Г., Тр. ИФ АН ЭССР, № 45, 5-42 (1976).
Институт физики
Академии наук Эстонской ССР
Поступила в редакцию
$21 / \mathrm{XII} 1979$

V. KORROVITS, M. TRUMMAL

\section{ADIABAATILINE ELEKTROKALORILINE EFEKT MONINGATES PARAELEKTRILISTES SUSTEEMIDES}

On arvutatud adiabaatilise elektrokalorilise efekti suurus ja lähendvalemid teatud mudeli jaoks. Ilmneb dipoolide spontaanse korrastumise tugev mõju süsteemides $\mathrm{KCl}: \mathrm{OH}^{-}$ja $\mathrm{RbCl}: \mathrm{CN}^{-}$madalatel temperatuuridel ja nõrgas elektriväljas, 
V. KORROVITS, M. TRUMMAL

\section{ADIABATIC ELECTROCALORIC EFFECT IN CERTAIN PARAELECTRIC SYSTEMS}

Magnitude and the approximation formulae of adiabatic electrocaloric effect (AEE) are calculated from the dipolar entropy. The system is modelled as an ideal gas of electric dipoles in cubic crystal with equilibrium orientations of $\vec{\mu}||<100>$. Tunneling transitions with turns of $\vec{\mu}$ to $90^{\circ}$ only are taken into account. Magnitude of AEE is computer calculated from the entropy equation. For $\mathrm{KCl}: \mathrm{OH}^{-}$this model gives quite satisfactory agreement with the experimental data on AEE. Discrepancy that rises at lower temperature and in weaker electric field is due to the chosen model which does not take into account local fields in the nearest proximity of impurities and the influence of impurity-impurity interaction to their orientation during the adiabatic depolarization. For certain intervals of parameters analytical approximation formulae are given for the magnitude of AEE: high temperature and weak electric field, low temperature and maximum field, low temperature and weak field approximations. The approximation of high temperature is in good agreement with experiment in $\mathrm{KCl}: \mathrm{OH}^{-}$ and $\mathrm{RbCl}: \mathrm{CN}^{-}$, it has the same form in the case of equilibrium orientations of $\vec{\mu}||<110>$ and $\vec{\mu}||<111>$. In two other approximations the coefficients vary. The results for low temperature and maximum field are quite close to experimental data in $\mathrm{KCl}: \mathrm{OH}^{-}$and $\mathrm{RbCl}: \mathrm{CN}^{-}$, in the latter case the experimental conditions are closer to the approximation intervals. There is satisfactory agreement between the low temperature and weak field approximation and experiment in $\mathrm{RbCl}: \mathrm{CN}^{-}$. In $\mathrm{KCl}: \mathrm{OH}^{-}$the polarization effects of local fields obviously dominate over weak external field. Derived approximation formulae for AEE may be useful for valuation of magnitude of the effect, as for the exact determination, quite laborious solvation of the entropy equation is needed. 